\title{
Recurrent Indeterminate Dendritic Cell Tumor of the Skin
}

\author{
Jin Woo Joo · Taek Chung \\ Yoon Ah Cho · Sang Kyum Kim \\ Department of Pathology, Severance Hospital, \\ Yonsei University College of Medicine, Seoul, \\ Korea
}

\author{
Received: September 14, 2017 \\ Revised: March 13, 2018 \\ Accepted: March 27, 2018

\section{Corresponding Author} \\ Sang Kyum Kim, MD, PhD \\ Department of Pathology, Severance Hospital, \\ Yonsei University College of Medicine, 50-1 \\ Yonsei-ro, Seodaemun-gu, Seoul 03722, Korea \\ Tel: +82-2-2228-6751 \\ Fax: +82-2-2227-7939 \\ E-mail: NICEKYUMI@yuhs.ac
}

Indeterminate dendritic cell tumor (IDCT) is a dendritic cell tumor that displays histologic features similar to those of Langerhans cells. The origin of the indeterminate cells may represent precursors of Langerhans cells or skin dendritic cells. IDCT is extremely rare, and tumor progression and predictive factors are not well known. Here, we report a case of a 61-year-old man who presented with a papule on his back and was finally diagnosed with IDCT based on histology and immunohistochemistry. The tumor recurred three months after surgical excision.

Key Words: Indeterminate dendritic cell tumor; Skin tumor; Recurrence
Indeterminate dendritic cell tumor (IDCT) is a proliferative disorder of indeterminate cells that has been reported since the 1980s. ${ }^{1,2}$ However, the origin of indeterminate cells is still debated. Indeterminate cells share histological, ultrastructural, and antigenic features of Langerhans cells, but they lack Birbeck granules on electron microscopic examination. ${ }^{1,2}$

The clinical course of IDCT is relatively good. However, associations between IDCT and other hematologic malignancies such as B-cell lymphoma and acute myeloid leukemia have been reported, ${ }^{3,4}$ and recurrent IDCT was reported in the oral mucosa, ${ }^{5}$ although there are no known prognostic factors. To our knowledge, recurrent IDCT in the skin after surgical resection is extremely rare. We herein describe a case of recurrent IDCT in the skin after surgical excision.

\section{CASE REPORT}

A 61-year-old man had started to develop a papule on his back approximately 2 years prior. After 1 year, the papule was removed by laser therapy. However, the patient continued to have multiple papules on his back, and he presented to our hospital. On physical examination, four asymptomatic erythematous papules were observed (Fig. 1A). The largest papule was approximately $2 \mathrm{~cm}$ in size (Fig. 1B). Since the patient's brother was diagnosed with acute leukemia, he had blood analyses which showed white blood cell count (WBC) of $3.4 \times 10^{9} / \mathrm{L}$, hemoglobin $14.7 \mathrm{~g} / \mathrm{L}$, and platelet count $99 \times 10^{9} / \mathrm{L}$. The patient underwent surgical excision of the papules. Gross examination of the largest nodule revealed a poorly defined infiltrative whitish mass $(1.8 \times 1.3 \mathrm{~cm})$ involving the dermis and subcutis, showing focal hemorrhage and necrosis (Fig. 1C).

Histological evaluation revealed the infiltration of large epithelioid cells with mild to moderate nuclear atypia and large, irregular folded or twisted nuclei with a solid growth pattern (Fig. 2A-C). Proliferative cells with abundant pale eosinophilic cytoplasm were noted. The lesion exhibited frequent mitotic figures (99/10 high-power field) and a few scattered multi-nucleated giant cells (Fig. 2D). Peritumoral lymphocytic infiltration was also observed, with the absence of eosinophilic infiltration. Immunohistochemistry showed that the tumor expressed S100 protein and CD1a, but not Langerin (Fig. 3A-C). The tumor focally expressed CD68 (Fig. 3D), but did not express CD3, CD20, CD21, CD30, CD31, CD34, CD45, CD99, CD163, MPO, cKit, Melan A, human melanoma black 45, or smooth muscle actin. The $\mathrm{Ki} 67$ labeling index was approximately $70 \%$. In situ hybridization for Epstein-Barr virus encoded RNA was negative 

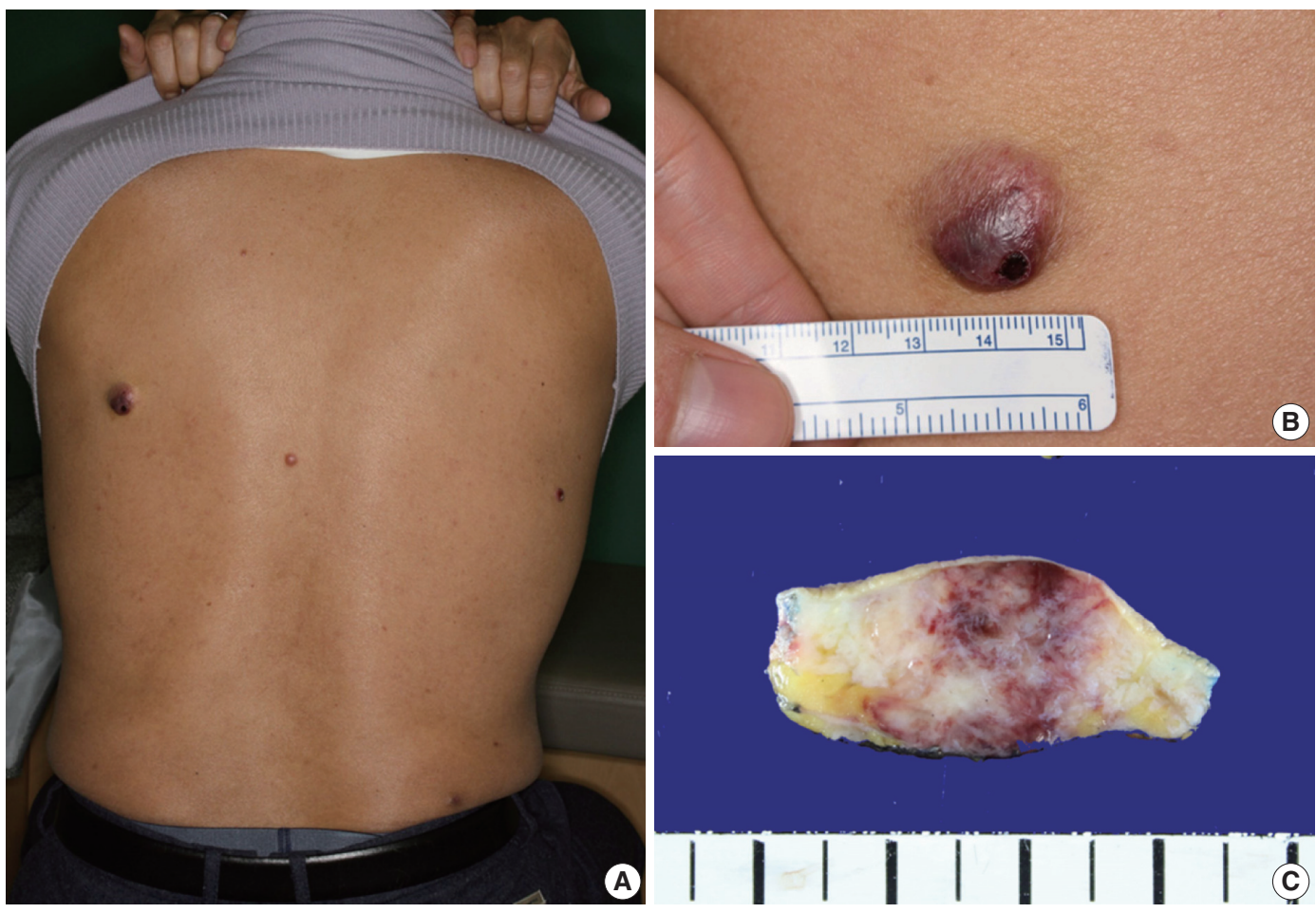

Fig. 1. Physical and gross findings. (A) Four erythematous papules on the patient's back. (B) The largest papule. (C) Gross examination of the largest nodule involving the dermis and subcutis.
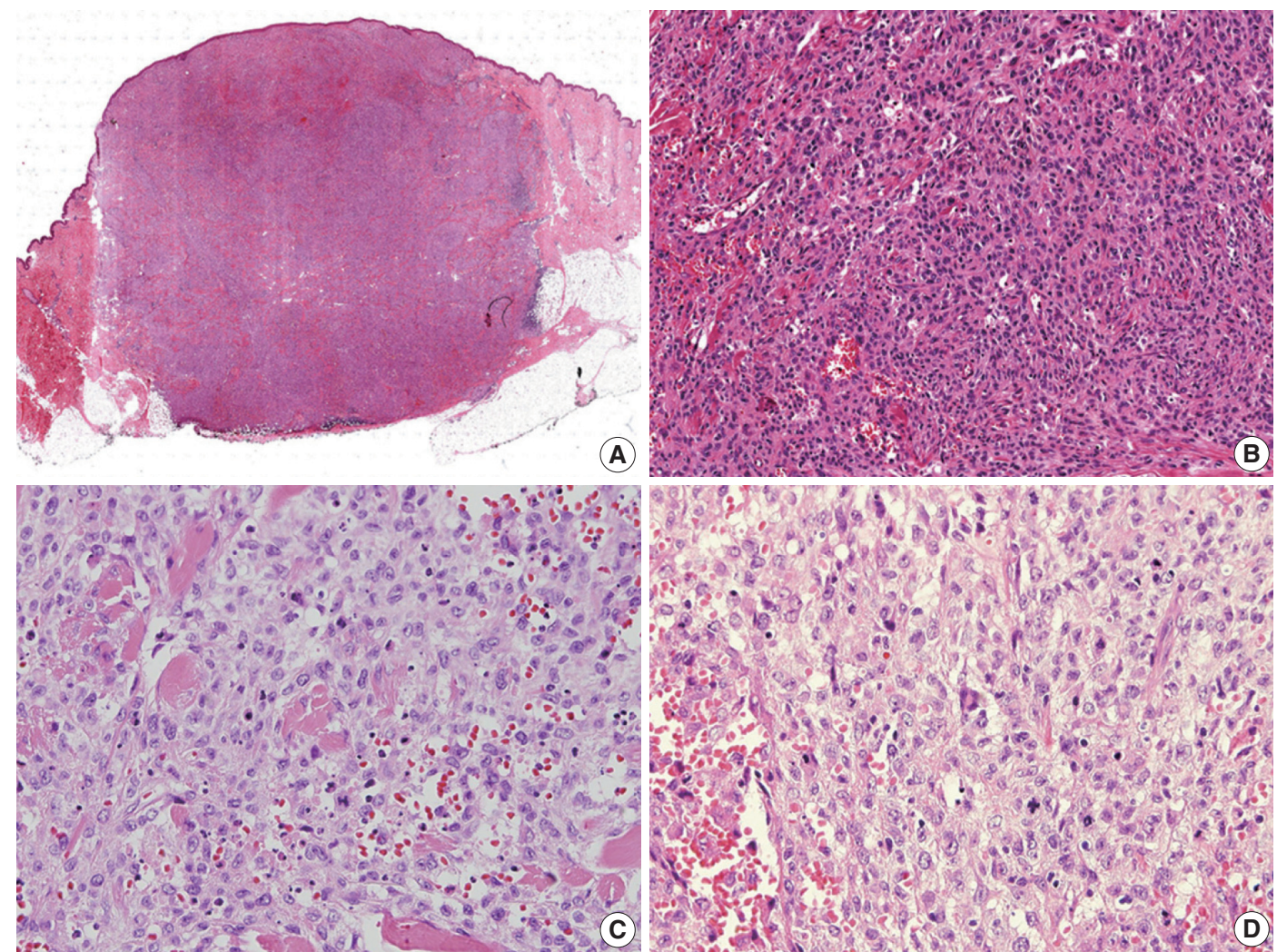

Fig. 2. Histologic findings. (A) Scan power view of the largest nodule. (B) A solid growth of epithelioid cells with infiltrative borders. (C) Epithelioid cells with moderate nuclear atypia and irregular folded nuclei. (D) Frequent mitoses and a few scattered multi-nucleated giant cells. 

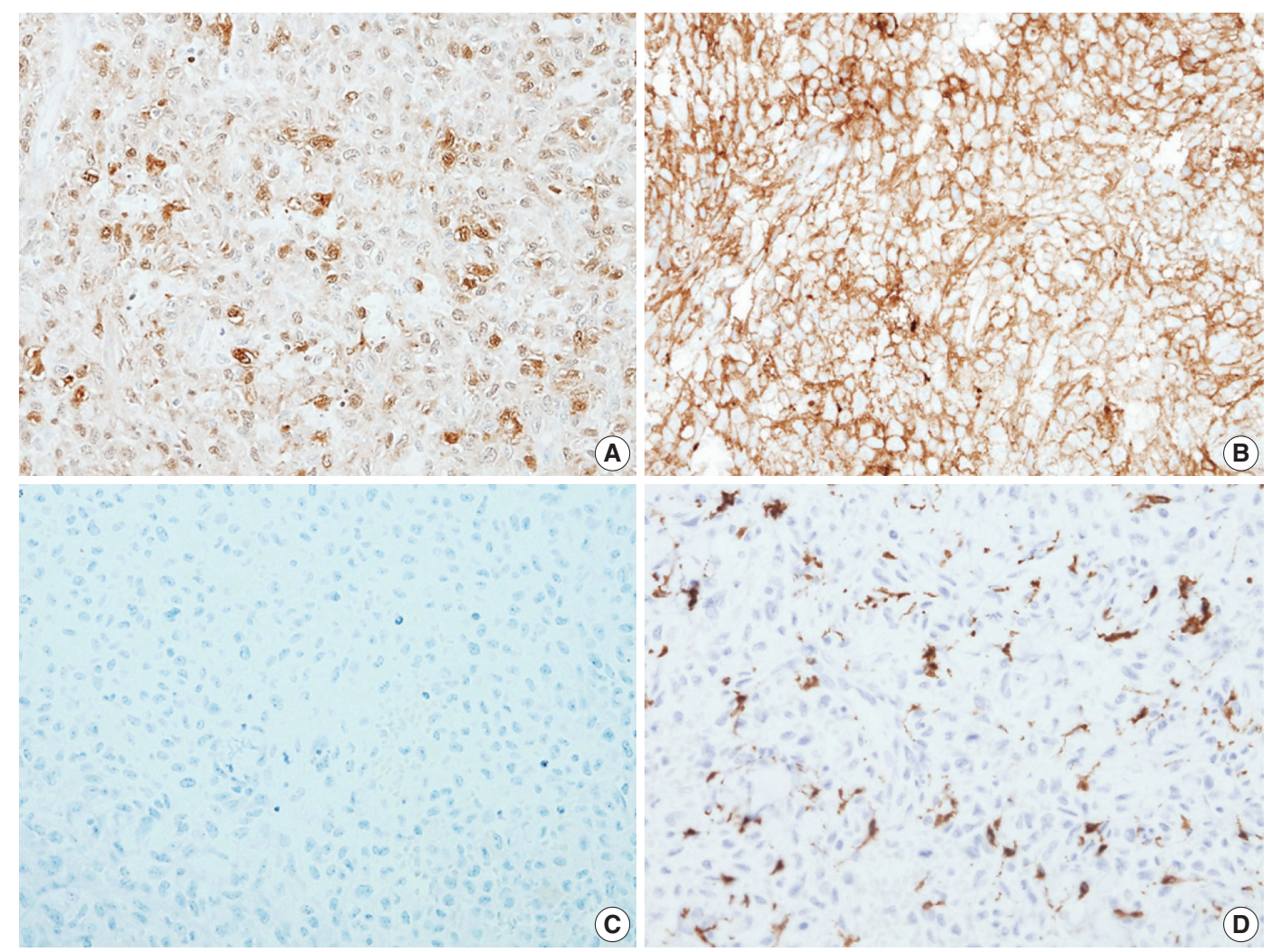

Fig. 3. The immunohistochemical stain results. Immunohistochemical panels of S100 (A), CD1a (B), Langerin (C), and CD68 (D).

and BRAF mutation was not identified by the pyrosequencing method. No ultrastructural examination was performed.

Two weeks after the operation, bone marrow examinations were performed because of the patient's low platelet count. A bone marrow aspiration and biopsy showed hypocellular marrow (about 20\%) with a slightly increased number of megakaryocytes. The proportion of megakaryocytes showing dysplastic features was increased, but did not meet the diagnostic criteria of myelodysplastic syndrome.

Two months after the bone marrow examinations, a $0.5 \mathrm{~cm}$ nodule was found on the patient's left buttock. The nodule was resected, and the tumor exhibited similar histological features as those of the previous specimens. Therefore, we diagnosed this tumor as recurrent IDCT. Three months later, multiple papulonodular skin lesions recurred on the patient's back and anterior chest. We observed these lesions without administering medical therapy. The patient was not examined with whole-body imaging technology for metastasis.

Hematology oncologists suggested that further whole body examinations should be performed because of the repeated recurrence. The latest blood examination results were within normal limits for WBC count, hemoglobin level, and platelet count
(WBC $5.81 \times 10^{9} / \mathrm{L}$, hemoglobin $11.2 \mathrm{~g} / \mathrm{dL}$, and platelet $120 \times$ $10^{9} / \mathrm{L}$ ). Chest X-ray did not reveal any suspicious metastatic lesions.

Authorization for the use of the case information and materials was obtained from the Institutional Review Board (IRB) of Yonsei University College of Medicine (4-2017-0669). Informed consent was waived because the IRB decided that this retrospective study presented minimal risk to the patient (risk level I).

\section{DISCUSSION}

IDCTs are believed to derive from cells with features similar to Langerhans and interdigitating cells. ${ }^{2}$ They have morphological and immunological similarities to normal Langerhans cells, but do not contain Birbeck granules. ${ }^{1,2}$ Clinically, IDCTs have most often been described in adults, who present with one or more lesions, commonly cutaneous, and rarely in the lymph nodes or spleen. ${ }^{1}$ The lesions are usually based in the dermis, occasionally with extension into the epidermis. The clinical course varies widely from spontaneous regression, to rapid progression or stable disease and to recurrence. ${ }^{1}$

Some recent studies suggest that Langerin (CD207) is quite a 
useful marker for tumors derived from Langerhans cells, with negative findings in reported IDCTs. ${ }^{6,7}$ Wang et al. ${ }^{8}$ reported on a Langerin-positive Langerhans cell sarcoma (LCS) that did not present with Birbeck granules on ultrastructural examination. ${ }^{8}$ This has occurred in some cases of LCS due to damage of Birbeck granules or poorly differentiated tumor cells. ${ }^{9}$ In conclusion, Langerin is a very specific marker for distinguishing IDCT from LCS. ${ }^{710}$ Our case showed typical histologic and immunophenotypic features including Langerin negativity. Therefore, this case is consistent with IDCT, even though an electron microscopic examination was not performed. Li et al. ${ }^{11}$ also studied IDCT in a 90-year-old man. Although they did not perform an ultrastructural examination, they used the pathologic evaluation and immunohistochemical analysis to diagnose IDCT. The tumor revealed aggressive histologic features such as central necrosis and a high Ki67 proliferation index (35\%). Moreover, multiple lymph node metastases were identified by computed tomography scan. The patient underwent radiation therapy, but died three months later due to circulatory and respiratory failure. The study suggested that old age, distant metastases, and a high Ki67 labeling index are potential prognostic factors. In our study, the Ki67 labeling index of the tumor cells was approximately $70 \%$.

Roh et al..$^{12}$ reported the first case of IDCT in Korea. The patient was a 29-year-old woman who had an erythematous nodule on the flank. Histologically, the nodule exhibited typical IDCT morphology without epidermotropism or eosinophilic microabscess. Unlike our case, this tumor did not present with tumor necrosis or frequent mitoses.

The proper treatment for IDCT has not been standardized because of its rarity. Various treatments of IDCT have been reported, and some therapies such as electron beam therapy and ultraviolet B phototherapy were successful. ${ }^{13,14}$ Since most IDCT cases are clinically benign, there is no requirement for aggressive treatment. ${ }^{15}$

A report by Ibrar et al. ${ }^{5}$ described recurrent multifocal IDCT involving the oral mucosa in an 86-year-old man. The patient initially had a skin lesion in his left temporal scalp. Three months later, multiple skin lesions were identified on the scalp. Finally, the tumors involved the mandible, buccal mucosa, and tongue with lymph node metastasis. This case exhibited a rapidly progressing clinical course and poor prognosis. In contrast, some cases in the literature reported spontaneous regression of IDCTs in skin lesions. ${ }^{16}$ In a study by Ratzinger et al. ${ }^{17}{ }^{17}$ most of the patients who were diagnosed with localized IDCT were completely cured after surgical excision.

Vasef $e t ~ a l .^{3}$ reported two cases of IDCT associated with lowgrade B-cell malignancies. These cases suggest that B-cell neo- plasms might be associated with several histiocytic and dendritic cell tumors. Furthermore, there is a possibility that histiocytic/ dendritic cells and B cells share a common precursor. ${ }^{2}$ Ventura et $a l^{18}$ reported a case of indeterminate cell histiocytosis that presented with normal findings on the hematologic examination by bone marrow biopsy at diagnosis. However, 7 months later, the blood examination represented hyperleukocytosis, anemia, and thrombocytopenia. The bone marrow biopsy revealed hypercellularity with extensive blast infiltration. The patient was finally diagnosed with acute monocytic leukemia.

According to a recent study, IDCT cells might contain the BRAF mutation. ${ }^{19}$ We performed BRAF mutation analysis in our case, and the result was negative. The recent study also discovered ETV3-NCOA2 translocations in a few cases of IDCT, ${ }^{19}$ suggesting that IDCTs may have a specific clonal neoplasm.

In our case, the microscopic findings revealed several aggressive features including frequent mitoses, a focal area of necrosis, and a high Ki67 labeling index. This corresponded to the patient's malignant clinical course. We could not rule out the possibility of lymph node metastasis or involvement of other organs.

\section{ORCID}

Sang Kyum Kim: https://orcid.org/0000-0003-0768-9923

\section{Conflicts of Interest}

No potential conflict of interest relevant to this article was reported.

\section{Acknowledgments}

The Basic Science Research Program through the National Research Foundation of Korea (NRF) and was funded by the Ministry of Education (grant number 2016R1D1A1B03931581).

\section{REFERENCES}

1. LeBoit PE, Burg G, Weedon D, Serasin A. Pathology and genetics of skin tumours. Lyon: IARC Press, 2006; 220.

2. Rezk SA, Spagnolo DV, Brynes RK, Weiss LM. Indeterminate cell tumor: a rare dendritic neoplasm. Am J Surg Pathol 2008; 32: 186876.

3. Vasef MA, Zaatari GS, Chan WC, Sun NC, Weiss LM, Brynes RK. Dendritic cell tumors associated with low-grade B-cell malignancies: report of three cases. Am J Clin Pathol 1995; 104: 696-701.

4. Vener C, Soligo D, Berti E, et al. Indeterminate cell histiocytosis in association with later occurrence of acute myeloblastic leukaemia. 
Br J Dermatol 2007; 156: 1357-61.

5. Ibrar W, Khurram M, Snower D. Recurrent multifocal indeterminate dendritic cell tumor involving oral mucosa: a rare case report. Am J Clin Pathol 2015; 144(Suppl 2): A316.

6. Zhao G, Luo M, Wu ZY, et al. Langerhans cell sarcoma involving gallbladder and peritoneal lymph nodes: a case report. Int J Surg Pathol 2009; 17: 347-53.

7. Chikwava K, Jaffe R. Langerin (CD207) staining in normal pediatric tissues, reactive lymph nodes, and childhood histiocytic disorders. Pediatr Dev Pathol 2004; 7: 607-14.

8. Wang C, Chen Y, Gao C, Yin J, Li H. Multifocal Langerhans cell sarcoma involving epidermis: a case report and review. Diagn Pathol 2012; 7: 99.

9. Ben-Ezra J, Bailey A, Azumi N, et al. Malignant histiocytosis X: a distinct clinicopathologic entity. Cancer 1991; 68: 1050-60.

10. Lau SK, Chu PG, Weiss LM. Immunohistochemical expression of Langerin in Langerhans cell histiocytosis and non-Langerhans cell histiocytic disorders. Am J Surg Pathol 2008; 32: 615-9.

11. Li Y, Wang TT, Zhang ZH, Wang L. Aggressive indeterminate dendritic cell tumor mimicking scalp angiosarcoma. Ann Dermatol 2017; 29: 614-7.

12. Roh J, Kim SW, Park CS. Indeterminate dendritic cell tumor: a case report of a rare Langerhans cell lineage disease. J Pathol Transl
Med 2016; 50: 78-81.

13. Malhomme de la Roche H, Lai-Cheong JE, Calonje E, Davies M, Morris S, Whittaker SJ. Indeterminate cell histiocytosis responding to total skin electron beam therapy. Br J Dermatol 2008; 158: 838-40.

14. Ishibashi M, Ouchi T, Tanikawa A, Ishiko A. Indeterminate cell histiocytosis successfully treated with ultraviolet B phototherapy. Clin Exp Dermatol 2008; 33: 301-4.

15. Sidoroff A, Zelger B, Steiner H, Smith N. Indeterminate cell histiocytosis: a clinicopathological entity with features of both $\mathrm{X}$ - and non-X histiocytosis. Br J Dermatol 1996; 134: 525-32.

16. Contreras F, Fonseca E, Gamallo C, Burgos E. Multiple self-healing indeterminate cell lesions of the skin in an adult. Am J Dermatopathol 1990; 12: 396-401.

17. Ratzinger G, Burgdorf WH, Metze D, Zelger BG, Zelger B. Indeterminate cell histiocytosis: fact or fiction? J Cutan Pathol 2005; 32: $552-60$.

18. Ventura F, Pereira T, da Luz Duarte M, Marques H, Pardal F, Brito C. Indeterminate cell histiocytosis in association with acute myeloid leukemia. Dermatol Res Pract 2010; 2010: 569345.

19. Emile JF, Abla O, Fraitag S, et al. Revised classification of histiocytoses and neoplasms of the macrophage-dendritic cell lineages. Blood 2016; 127: 2672-81. 